\title{
Analytic solution for two-dimensional heat equation for an ellipse region
}

\author{
Nurcan Baykus Savasaneril ${ }^{1}$ and Havva Delibas ${ }^{2}$ \\ ${ }^{1}$ Vocational School, Dokuz Eylul University, Izmir, Turkey \\ ${ }^{2}$ Department of Mathematics, Faculty of Sciences, University of Selcuk, Konya, Turkey \\ Received: 10 June 2015, Revised: 24 June 2015, Accepted: 5 August 2015 \\ Published online: 1 January 2016.
}

\begin{abstract}
In this study, an altenative method is presented for the solution of two-dimensional heat equation in an ellipse region. In this method, the solution function of the problem is based on the Green, and therefore on elliptic functions. To do this, it is made use of the basic consepts associated with elliptic integrals, conformal mappings and Greenfunctions.
\end{abstract}

Keywords: Heate quation, dirichlet problem, elliptic function, elliptic integral, Green function.

\section{Introduction}

Laplace's equation is one of the most significant equations in physics. It is the solution to problems in a wide variety of fields including thermodynamics and electrodynamics. Today, the theory of complex variables is used to solve problems of heat flow, fluid mechanics, aerodynamics, electromagnetic theory and practically every other field of science and engineering. A broad class of steady-state physical problems can be reduced to finding the harmonic functions that satisfy certain boundary conditions. The Dirichlet problem for theLaplace equation is one of the above mentioned problems.

The Dirichlet problem is to find a function $U(z)$ that is harmonic in a bounded domain $D \subset R^{2}$, is continuous up to the boundary $\partial D$ of $D$, assumes the specified values $U_{0}(z)$ on the boundary $\partial D$, where $U_{0}(z)$ is a continuous function on $\partial D$, and can be formulated as

$$
\nabla^{2} U=0, \quad z \in D, \quad \underset{z \in \partial D}{U}=U_{0}(z)
$$

Here, for a point $(x, y)$ in the plane ${ }^{2}$, one takes the complex notation $z=x+i y, U(z)=U(x, y)$ and $U_{0}(z)=U_{0}(x, y)$ are real functions and $\nabla^{2}=\frac{\partial^{2}}{\partial x^{2}}+\frac{\partial^{2}}{\partial y^{2}}$ is the Laplace operator. Similarly the Dirichlet problem for the Poisson equation can be formulated as

$$
\nabla^{2} U=h(z), \quad z \in D, \underset{z \in \partial D}{U \mid}=U_{0}(z)
$$

Brovar et al. [1] investigated the relation between the Dirichlet problem and the Cauchy problem. Sezer [2] developed a new method for the solution of Dirichlet problem. Lanzara [3] studied Dirichlet problem for second degree elliptic linear equations with limited and measurable coefficients. The dependence upon variations of problem data of the solution of two-dimensional Dirichlet boundary value problem for simply connected regions was investigated [4]. Han and Hasebe 
[5] derived Green's function for a thermomechanical mixed boundary value problem of an infinite plane with an elliptic hole under a pair of heat source and sink. In another study, Han and Hasebe [6] also reviewed Green's functions for a point heat source in various thermoelastic boundary value problems for an infinite plane with an inhomogeneity. Green function of the Dirichlet problem for the Laplace differential equation in a rectangular domain was expressed in terms of elliptic functions and the solution of the problem was based on the Green function and therefore on elliptic functions by Kurtet al. [7]. Hsiao and Saranen [8] showed an equivalence between the weak solution and the various boundary integral solutions, and described a coupling procedure for an exterior initial boundary value problem for the nonhomogeneous heat equation. The problem of the one-dimensional heat equation with nonlinear boundary conditions was studied by Tao [9]. Hansen [10] studied a boundary integral method for the solution of the heat equation in an unbounded domain D in $\mathrm{R}^{2}$. The application of spectral methods for solving the one-dimensional heat equation was presented by Saldana et al. [11]. Al-Najem et al. [12] estimated the surface temperature in two-dimensional steady-state in a rectangular region by two different methods, the singular value decomposition with boundary element method and the least-squares approach with integral transform method. The Green function of the Dirichlet problem for the Laplace differential equation in a triangle region was expressed in terms of elliptic functions and the solution of problem was based on the Green function, and therefore on elliptic functions by Kurt and Sezer [13]. Green function of the two-dimensional heat equation in a square region was expressed in terms of elliptic functions and the solution of the problem was based on the Green function and therefore on elliptic functions by Kurt [14]. Least Square Method (LSM), Collocation Method (CM) and a new approach which is called Akbari-Ganji's Method (AGM) are applied to solve the nonlinear heat transfer equation of fin with power-law temperature-dependent both thermal conductivity and heat transfer coefficient by Ledari et al. [15].

As it is known, the solution of the Dirichlet problem by the method of separation of variables may be obtained only for a restricted class of domains $D$ with a sufficiently simple boundary $\partial D$. The conformal mappings yield a sufficiently universal algorithm for the solution of the Dirichlet problem for two-dimensional domains. These permit constructing a Green function of the Dirichlet problem for the Laplace (and Poisson) equation in a Dconformally mapped onto the unit circle or upper half-plane, and cannot be obtained in terms of elliptic functions.

Our purpose in this paper is, first, to determine the analytic function which maps the ellipse domain $D$ onto the upper half-plane or the unit circle in terms of elliptic functions using the Schwarz-Christoffel transformation and conformal mapping, and then, to find the solution of the Dirichlet problem for the ellipse domain in terms of elliptic functions, by means of the relation between the obtained analytic function and the Green function.

\section{Elliptic integrals and functions}

The integral

$$
\int_{0}^{t} \frac{d \tau}{\sqrt{\left(1-\tau^{2}\right)\left(1-k^{2} \tau^{2}\right)}}=\int_{0}^{u} d u_{1}=u=s n^{-1}(t, k)=F(\varphi, k) t=\sin \varphi
$$

is called the normal elliptic integral of the first kind, where $k,(0<k<1)$ is any number. When $t=1$, Eq. (3) is said to be complete and becomes

$$
\int_{0}^{t} \frac{d \tau}{\sqrt{\left(1-\tau^{2}\right)\left(1-k^{2} \tau^{2}\right)}}=\int_{0}^{K} d u_{1}=F\left(\frac{\pi}{2}, k\right) \equiv K(k) \equiv K
$$




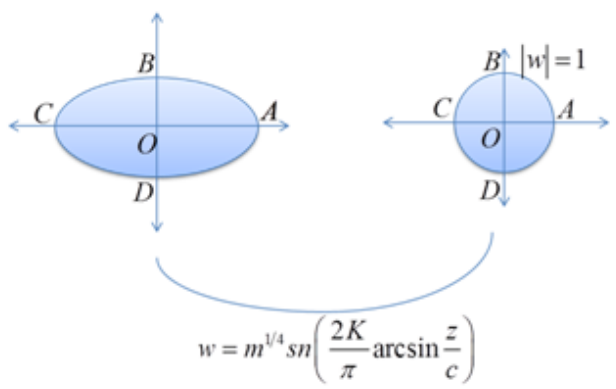

Fig. 1: The ellipse with two slits drawn.

or

$$
\int_{0}^{t} \frac{d \tau}{\sqrt{\left(1-\tau^{2}\right)\left(1-k^{\prime 2} \tau^{2}\right)}}=\int_{0}^{K^{\prime}} d u_{1}=F\left(\frac{\pi}{2}, k^{\prime}\right) \equiv K^{\prime}(k) \equiv K\left(k^{\prime}\right) \equiv K^{\prime}
$$

Here, the number $k$ is the modulus and $k^{\prime},\left(0<k^{\prime}<1\right)$ is the complementary modulus, such that $k^{\prime 2}=1-k^{2}$. If $k=0$ in Eq. (3), one finds that $u=\sin ^{-1} t$ or $t=\sin u$. When $k \neq 0$, the integral (3) is denoted by $u=s n^{-1}(t, k)$ or briefly $u=s n^{-1} t$ or $t=s n u$. The function sn $u$ is called Jacobian elliptic function. The other Jacobian elliptic functions can be defined by $c n(u, k)=\left(1-k^{2}\right)^{1 / 2}$.

\section{The conform mapping of an ellipse domain}

Let apply the transformation $\zeta_{1}=\frac{z}{c}$ to carry the ellipse onto the unit circle $|w|<1$.

The ellipse with two slits drawn in Fig.1. is mapped onto a rectangle in the $\zeta_{2}-$ plane by means of $\zeta_{2}=\arcsin \zeta_{1}$. Let $\frac{\pi}{2}=K$ and $\arcsin h\left(\frac{b}{c}\right)=\frac{K^{\prime}}{2}$, that is $K^{\prime}=\left(\frac{4 K}{\pi}\right) \arcsin h\left(\frac{b}{c}\right)$. With $\zeta=\left(\frac{2 K}{\pi}\right) \zeta_{1}, \quad w_{1}=s n \zeta$, the horizontal sides are mapped onto arcs of a circle and the vertical sides onto the edges of two cuts. In this way, each cut in thez- plane corresponds to a cut in the $w$ - plane. Consequently, these cuts can be sealed. In order to obtain $m$ from $K$ and $K^{\prime}$, note that

$$
q=\exp \left(\frac{-K^{\prime} \pi}{K}\right)=\exp \left(-4 \arcsin h \frac{b}{c}\right)=\frac{c^{4}}{(b+a)^{4}}=\frac{(a-b)^{2}}{(a+b)^{2}}
$$

The conformal mapping of the ellipse given in the $z$-plane onto the unit circle $|w|<1$ in the $w$ - plane can be written as [16]

$$
w=m^{\frac{1}{4}} \operatorname{sn}\left(\frac{2 K}{\pi} \arcsin \frac{z}{c}\right)
$$

\section{Determination of Green function}

Green function $G(z, \zeta)$ of the Dirichlet problem for the Laplace equation in the domain $D$ is defined by

$$
G(z, \zeta)=\frac{1}{2 \pi} \ln |z-\zeta|+g(z, \zeta) \quad z \in D, \zeta \in D
$$


where $g$ is a harmonic function in $D$ for each $\zeta \in D$ and $g(z, \zeta)=-1 /(2 \pi) \ln |z-\zeta|$ then $G(z, \zeta)=0$, for each $z \in \partial D, z=x+i y$ and $\zeta=\xi+i \eta$.

When the domain $D$ is simply connected, the determination of the mentioned Green function can be reduced to the problem of determining an analytic function which specifies a mapping of $D$ onto the upper half-plane $\operatorname{Im} W>0$ or the unit circle $|w|<1$. This is so because, $W=F(z)$ is an analytic functi on which maps the domain $D$ in the $z$-plane onto the upper half-plane of the $W$ - plane, with $F^{\prime}(z) \neq 0$ in $D$ then the mapping is one-to-one.

$$
G=\frac{1}{2} \ln \left|\frac{F(z)-F(\zeta)}{F(z)-\overline{F(\zeta)}}\right|, z=x+i y, \zeta=\xi+i \eta
$$

and, if the analytic function $W=F(z)$ maps $D$ onto the unit circle $|w|<1$, then the Green function of the Dirichlet problem for the Laplace operator in $D$ becomes

$$
G(z, \zeta)=\frac{1}{2 \pi} \ln |w(z-\zeta)|, W(z, \zeta)=\frac{F(z)-F(\zeta)}{1-F(z) \overline{F(\zeta)}}
$$

Consequently, if one takes D as the elliptic $A(K, 0), B\left(0, K^{\prime}\right), C(-K, 0), D\left(0,-K^{\prime}\right)$ the from Eq.(6) and (9), the Green function for the cut-ring is found in the from

$$
G=\frac{1}{2 \pi} \ln \left|\frac{m^{\frac{1}{4}} \operatorname{sn}\left(\frac{2 K}{\pi} \arcsin \frac{z}{c}\right)-m^{\frac{1}{4}} \operatorname{sn}\left(\frac{2 K}{\pi} \arcsin \frac{\zeta}{c}\right)}{1-m^{\frac{1}{4}} \operatorname{sn}\left(\frac{2 K}{\pi} \arcsin \frac{z}{c}\right) \cdot m^{\frac{1}{4}} \operatorname{sn}\left(\frac{2 K}{\pi} \arcsin \frac{\zeta}{c}\right)}\right| .
$$

\section{The solution of the Dirichlet problem}

The solution of the Dirichlet problem for the Poisson equation (2) in $D$ can be obtained as

$$
U(z)=\int_{D} \int G(z, \zeta) h(\zeta) d \xi d \eta+\int_{\partial D} \frac{G(z, \zeta)}{\partial n} U_{0}(\zeta)|d \zeta| .
$$

Where $G$ is the Green function for the domain $D$ and $\partial / \partial n$ denotes differentiation along an outward normal to the boundary $\partial D$ of $D$ with respect to $\zeta$.

Taking the domain $D$ as the ellipse $A(K, 0), B\left(0, K^{\prime}\right), C(-K, 0), D\left(0,-K^{\prime}\right)$ and the boundary $\partial D$ of $D$ as the circumference $\partial D=\widehat{A B} \cup \widehat{B C} \cup \widehat{C D} \cup \widehat{D A}$, one may write the conditions

(a) $\eta=\frac{K^{\prime}}{K} \sqrt{K^{2}-\xi^{2}}, \quad d \eta=\frac{K^{\prime}}{K} \frac{-\xi}{\sqrt{K^{2}-\xi^{2}}} d \xi,|d \zeta|=\sqrt{\frac{K^{2}\left(K^{2}-\xi^{2}\right)+\left(K^{\prime}\right)^{2} \xi^{2}}{K^{2}\left(K^{2}-\xi^{2}\right)}} d \xi, 0 \leq \xi \leq K$, on $\widehat{A B}$,

(b) $\eta=\frac{K^{\prime}}{K} \sqrt{K^{2}-\xi^{2}}, \quad d \eta=\frac{K^{\prime}}{K} \frac{-\xi}{\sqrt{K^{2}-\xi^{2}}} d \xi, \quad|d \zeta|=\sqrt{\frac{K^{2}\left(K^{2}-\xi^{2}\right)+\left(K^{\prime}\right)^{2} \xi^{2}}{K^{2}\left(K^{2}-\xi^{2}\right)}} d \xi,-K \leq \xi \leq 0$ on $\widehat{B C}$,

(c) $\eta=-\frac{K^{\prime}}{K} \sqrt{K^{2}-\xi^{2}}, \quad d \eta=\frac{K^{\prime}}{K} \frac{\xi}{\sqrt{K^{2}-\xi^{2}}} d \xi,|d \zeta|=\sqrt{\frac{K^{2}\left(K^{2}-\xi^{2}\right)+\left(K^{\prime}\right)^{2} \xi^{2}}{K^{2}\left(K^{2}-\xi^{2}\right)}} d \xi,-K \leq \xi \leq 0$ on $\widehat{C D}$,

(d) $\eta=-\frac{K^{\prime}}{K} \sqrt{K^{2}-\xi^{2}}, \quad d \eta=\frac{K^{\prime}}{K} \frac{\xi}{\sqrt{K^{2}-\xi^{2}}} d \xi, \quad|d \zeta|=\sqrt{\frac{K^{2}\left(K^{2}-\xi^{2}\right)+\left(K^{\prime}\right)^{2} \xi^{2}}{K^{2}\left(K^{2}-\xi^{2}\right)}} d \xi, 0 \leq \xi \leq K$ on $\widehat{D A}$,

Thus, from Eq. (16), the solution of Eq. (2) in the above rectangle becomes

$U(z)=\int_{-K}^{K} \int_{-K^{\prime}}^{K^{\prime}} G(z, \zeta) h(\zeta) d \xi d \eta+\int_{0}^{K}\left[G_{\xi}^{2}(z, \zeta)+G_{\eta}^{2}(z, \zeta)\right]^{1 / 2} U_{0}\left(\xi, \frac{K^{\prime}}{K} \sqrt{K^{2}-\xi^{2}}\right) \sqrt{\frac{K^{2}\left(K^{2}-\xi^{2}\right)+\left(K^{\prime}\right)^{2} \xi^{2}}{K^{2}\left(K^{2}-\xi^{2}\right)}} d \xi$ 


$$
\begin{aligned}
& +\int_{-K}^{0}\left[G_{\xi}^{2}(z, \zeta)+G_{\eta}^{2}(z, \zeta)\right]^{1 / 2} U_{0}\left(\xi, \frac{K^{\prime}}{K} \sqrt{K^{2}-\xi^{2}}\right) \sqrt{\frac{K^{2}\left(K^{2}-\xi^{2}\right)+\left(K^{\prime}\right)^{2} \xi^{2}}{K^{2}\left(K^{2}-\xi^{2}\right)}} d \xi \\
& -\int_{-K}^{0}\left[G_{\xi}^{2}(z, \zeta)+G_{\eta}^{2}(z, \zeta)\right]^{1 / 2} U_{0}\left(\xi,-\frac{K^{\prime}}{K} \sqrt{K^{2}-\xi^{2}}\right) \sqrt{\frac{K^{2}\left(K^{2}-\xi^{2}\right)+\left(K^{\prime}\right)^{2} \xi^{2}}{K^{2}\left(K^{2}-\xi^{2}\right)}} d \xi \\
& -\int_{0}^{K}\left[G_{\xi}^{2}(z, \zeta)+G_{\eta}^{2}(z, \zeta)\right]^{1 / 2} U_{0}\left(\xi,-\frac{K^{\prime}}{K} \sqrt{K^{2}-\xi^{2}}\right) \sqrt{\frac{K^{2}\left(K^{2}-\xi^{2}\right)+\left(K^{\prime}\right)^{2} \xi^{2}}{K^{2}\left(K^{2}-\xi^{2}\right)}} d \xi
\end{aligned}
$$

In the case of $h(z)=0$, the solution of the Dirichlet problem for the Laplace differential equation (1) in the above rectangle is obtained in terms of elliptic functions as,

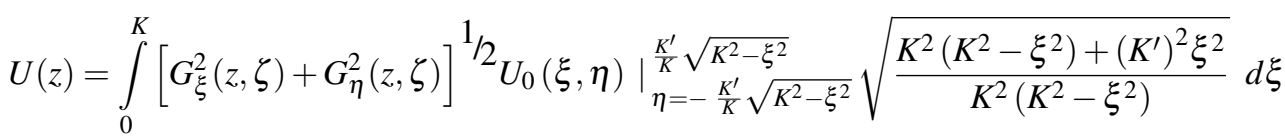

$$
\begin{aligned}
& +\left.\int_{-K}^{0}\left[G_{\xi}^{2}(z, \zeta)+G_{\eta}^{2}(z, \zeta)\right]^{1 / 2} U_{0}(\xi, \eta)\right|_{\eta=-\frac{K^{\prime}}{K} \sqrt{K}} ^{\frac{K^{\prime}}{K^{2}-\xi^{2}}} \sqrt{\frac{K^{2}\left(K^{2}-\xi^{2}\right)+\left(K^{\prime}\right)^{2} \xi^{2}}{K^{2}\left(K^{2}-\xi^{2}\right)}} d \xi,
\end{aligned}
$$

where the Green function $G$ is defined by

$$
G=\frac{1}{2 \pi} \ln \left|\frac{m^{\frac{1}{4}} \operatorname{sn}\left(\frac{2 K}{\pi} \arcsin \frac{z}{c}\right)-m^{\frac{1}{4}} \operatorname{sn}\left(\frac{2 K}{\pi} \arcsin \frac{\zeta}{c}\right)}{1-m^{\frac{1}{4}} \operatorname{sn}\left(\frac{2 K}{\pi} \arcsin \frac{z}{c}\right) \cdot m^{\frac{1}{4}} \operatorname{sn}\left(\frac{2 K}{\pi} \arcsin \frac{\zeta}{c}\right)}\right|, z=x+i y, \zeta=\xi+i \eta,
$$

here Green function $G(z, \zeta)$ expressed in the form of elliptic functions. $K(k)$ and $K^{\prime}(k)$ integral boundaries are modular full elliptic integrals and are given for every given value of $\mathrm{k}(0<\mathrm{k}<1)$ constant; which can be calculated by the help of given tables[19].

\section{Discussion}

The proposed new method are used to find the solution of the Dirichlet Problem in 2-D. The method of conformal mapping is a more flexible and far-reaching tool for the Laplace equation in the plane. In a certain sense conformal mapping provides the analogue for elliptic differential equations of the method of characteristics developed for hyperbolic differential equations. The most important advantage of present method is that the result is obtained in terms of elliptic functions; because expressing the result in terms of elliptic functions facilitates many physics and engineering problems. Also, our method may be used for the solution of similar problems in physics and engineering based on another domains.

\section{References}

[1] V.V. Brovar, Z.S. Kopeikina, M.V. Pavlova, Solution of the Dirichlet and Stokes exterior boundary problems for the Earth.sellipsoid, J. Geodesy 74 (2001) 767.772.

[2] M. Sezer, Solution of Dirichlet Problem in Terms of Elliptic Functions, Yıldız University Dergisi 4 (1992) 1.4.

[3] F. Lanzara, Numerical approximation of eigenvalues and of Green's operator for an elliptic boundary value problem, Calcolo 35 (1998) 63.92. 
[4] A. Marshakov, P. Wiegmann, A. Zabrodin, Integrable structure of the Dirichlet boundary problem in two dimensions, Commun. Math. Phys. 227 (2002) 131.153.

[5] J.J. Han, N. Hasebe Green's function for a thermomechanical mixed boundary value problem of an infinite plane with an elliptic hole , Journal of Thermal Stresses 2001; 24(9):903-916.

[6] J.J. Han, N. Hasebe, Green's function of point heat sources in various thermoelastic boundary value problems, J. Thermal Stresses 25 (2002) 153.167.

[7] N. Kurt, M. Sezer, A. Çelik, Solution of Dirichlet problem for a rectangular region in terms of ellipticfunctions, J. Comput. Math. 81 (2004) 1417.1426.

[8] G.C. Hsiao, J. Saranen, Boundary integral solution of thetwo-dimensionalheatequation, Math. MethodsAppl. Sci. 16 (2) (1993) 87.114.

[9] L.N. Tao, The analyticity of solution of the heat equation with nonlinear boundary conditions, Quart. Mech. Appl. Math. 38 (1985) 447.459.

[10] O. Hansen, On a boundary integral method for the solution of the heat equation in unbounded domains with non smooth boundary, J. Integral Equation Appl. 12 (2000) 385.420.

[11] J.G. Saldana, J.A.J. Bernal, C.G. Torres, P.Q. Diez, Numerical solution for the one dimension heat equation by a pseudo-spectral discretization technique, Cienti.ca Instituto Politecnico Nacional, vol. 10, 2006, pp. 3.8.

[12] N.M. Al-Najem, A.M. Osman, M.M. El-Refaee, K.M. Khanafer, Two- dimensional steady-state in verse heatc onduction problems, Int. Commun. Heat Mass Transfer 25 (1998) 541.550.

[13] N. Kurt, M. Sezer, Solution of Dirichlet problem for a triangle region in terms of elliptic functions, Appl.Math. Comput. 182 (2006) 73.78.

[14] N.Kurt, Solution of the two-dimensional heat equation for a square in terms of elliptic function, Journal of the Franklin institute, 345, (2008) 303-317.

[15] S.T. Ledari, H. Mirgolbabaee and D. D. Ganji,Heat transfer analysis of afin with temperature dependent thermalconductivity and heat transfer coefficient, New Trends in Mathematical Sciences, 2015.

[16] G. Moretti, Functions of Complex Variable, Prentice-Hall, NJ, 1964.

[17] F.R. Hildebrand, Advanced calculusfor Applications, Prentice-HallInc. Englewood Clis, NJ,1976.

[18] P.R. Garabedian, Partial Differential Equations, John Wiley and Sons Inc.,New York,1964.

[19] P. F. Byrd and M.D.Friedman, Handbook of Elliptic Integrals for Engineers and Physicists, Lange Maxvell and Springer Ltd. London, New York, 1954 